\title{
The use of time-out in controlling hallucinatory behavior in a mentally retarded adult
}

\author{
DONALD P. HERRON and DIANE DeARMOND \\ University of Missouri-Kansas City, Kansas City, Missouri 64110
}

\begin{abstract}
A time-out procedure was used to decelerate hallucinatory behavior in a mentally retarded adult. Time-out consisted of removing the subject from the room in which the behavior occurred and then placing him in a corner for a duration of $5 \mathrm{~min}$. The results show that time-out effectively decelerated hallucinations after 1 week and extinguished the behavior completely after 9 weeks. The notable factor of the research is that it provides a method for treating psychotic behavior that has a minimal amount of interference with nonsymptomatic behavior.
\end{abstract}

Hallucinatory behavior has concerned psychologists for decades, but very little research has been conducted concerning this matter in general, and much less in the case of the mentally retarded. Reed's (1972) review of the literature shows that research on hallucinatory behavior falls into three basic categories: (1) those studies concerned with the deliberate experimental elicitation of responses to hallucinatory stimuli: (2) those studies where "hallucinatory" experiences have been reported among the effects of some experimental situation; and (3) those studies that have considered the cognitive characteristics of people who confuse their imagery with external reality. Very few studies based on operant principles have dealt with the treatment of hallucinatory behavior (Alumbaugh, 1971; Haynes \& Geddy, 1973; Rutner \& Bugle, 1969). Davidson, (1969) has reported that hallucinatory behavior tends to interfere with competent social functioning. This may be extremely critical for the mentally retarded, in that it is a competing factor with the principles of normalization (Wolfensberger, 1971), and thus impedes developmental training. It has been reported (Boston \& Bailey, 1969) that disruptive and aggressive behavior can be modified with the use of

time-out in mentally retarded adults. Also, Haynes and Geddy (1973) demonstrated that hallucinations could be suppressed in a female schizophrenic through the use of time-out. Thus, the present study attempted to use time-out contingencies in order to decelerate visual and auditory hallucinations in a mentally retarded adult.

\section{METHOD}

\section{Subject}

The subject was a 23-year-old Caucasian male, diagnosed as mentally retarded, with a full scale IQ of 51 on the Wechsler Adult Intelligence Scale. The subject had cerebral palsy with right hemiparesis, a sensorineural hearing impairment, and epilepsy, with the seizures under the control of diphenylhydantoin sodium capsules, $100 \mathrm{mg}$, two times daily. The subject also had a history of hallucinatory behavior.

\section{Response Definition}

The recorded pinpoint was defined as follows: Hallucinatory behavior was any verbal behavior that (1) had an abnormally high degree of competition with strongly reinforced nonsymptomatic behavior, (2) was independent of direct reinforcement (Lindsley, 1963), and (3) was without corresponding sensory stimuli in the appropriate modality. Examples of such behavior were moving the furniture out of the building because it was on fire (when it actually was not), attempting to put out the fire, telephoning the fire department, and, finally, talking to people who were not there.

\section{Procedure}

Prior to the initiation of the experimental procedures, a 4-day baseline was obtained. After this period, the treatment phase was introduced. Staff members were instructed to place the subject in time-out for a duration of 5 min each time he exhibited hallucinatory behavior. Time-out consisted of placing the subject in any corner, in any room other than the room in which the behavior was exhibited, with his face toward the wall. The subject was required to spend the total $5 \mathrm{~min}$ in time-out without any verbalization and without leaving the corner before the period ended. If such behaviors were emitted, the time-out period was started over.

When time-out was administered, the staff member would approach the subject and say one of the following phrases, depending on the characteristics of the hallucination. For an auditory hallucination, the staff member would say, "You have to stand in the corner because you were talking to yourself. The person to whom you were talking is not in your presence." For a visual hallucination, the staff member would ask the subject why he was behaving in such a manner and, if the subject's reply was consistent with his behavior and the reply and the behavior fit the definition of a hallucination, the staff member would reply, "What you have just said makes no sense, there is no basis for your thoughts or actions, you must go stand in the corner." After repeating one of the above phrases, the staff member led the subject to a corner in another room and set a timer for $5 \mathrm{~min}$.

\section{Data Collection}

The number of hallucinations per day was recorded and the rate per day was determined by dividing by a $1,000-\mathrm{min}$ day. The rates were then charted on six-cycle semilogarithmatic graph paper. The total days of recording werc 128 .

\section{Reliability}

Interobserver agreement was assessed by comparing the independent records of two observers who simultaneously recorded the behavior. Reliability checks were made on $42 \%$ 
of the first 24 days of observation and $25 \%$ of the total days. Interobserver agreement was calculated for the occurrence of the behavior. Reliability for the behavior was calculated by dividing agreements between observers by agreements plus disagreements and multiplying by 100 .

\section{RESULTS}

Agreement among staff members that the subject was hallucinating was $100 \%$ for the first 24 days of the experiment and $99.6 \%$ for the total observation period.

The results of the experiment are presented in Figure 1, which identifies reported hallucinatory behavior on a daily basis. During the baseline phase, the behavior accelerated by $\times 10$ movements per week $(\mathrm{m} / \mathrm{m} / \mathrm{w})$. The subject hallucinated about one time every $10 \mathrm{~min}$ for the first 3 days of observation, and on Day 4 he hallucinated about once every $4 \mathrm{~min}$.

Beginning with Day 5 of observation, the treatment period was introduced. Hallucinatory behavior dropped only slightly the first day. On Day 6 , the subject had only one hallucination during the entire day. During Day 8 of the recording period, no hallucinatory behavior was observed by the staff.

It took 6 days of time-out before the behavior decelerated to zero on a steady basis. From Day 1 of the treatment period, hallucinatory. behavior decelerated by $\div 1,000 \mathrm{~m} / \mathrm{m} / \mathrm{w}$. From the time the behavior reached a zero rate on a steady basis to 14 days later, the subject had seven more hallucinations. Six weeks after this period, the subject had eight more hallucinations, which may be attributed to a period of spontaneous recovery. After this period, no hallucinatory behavior was exhibited for over 9 weeks.

In summary, hallucinatory behavior decelerated from a rate of approximately one hallucination every $10 \mathrm{~min}$ during the baseline phase to a rate of zero on a steady basis after 1 week of treatment. In less than 9 weeks, hallucinatory behavior was extinguished completely.

\section{DISCUSSION}

The significant aspect of this research was to reduce the rate of psychotic behavior in a mentally retarded individual. Time-out appeared to be an effective mechanism in reducing hallucinatory behavior in a short span of time.

It would seem that this study has less variability than the Haynes and Geddy (1973) research, in that it provided continuous recording of the behavior. Lindsley (1963) has stated that we need to investigate the behavior of individual patients under a given experimental condition at least long enough to insure that any observed behavioral changes are not due to intermittently recurring psychotic phases. Another aspect of this discussion is that the patient may learn to avoid the individuals observing and continue to emit hallucinatory behavior in their absence. Further investigation of this problem is needed, but it appears that continuous observation and treatment will help the patient learn that the behavior is inappropriate in all settings.

The notable factor of this research is that it provided the therapist with a means of treating hallucinatory behavior without major interference with nonsymptomatic behavior.

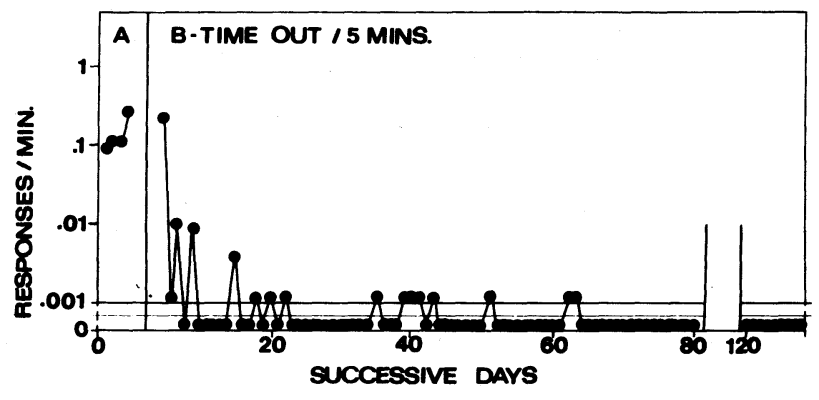

Figure 1. The daily rate of hallucinatory behavior across (A) baseline and (B) treatment conditions. The behavior decelerated from over .1 movements $/ \mathrm{min}$ during the baseline phase to 0.0 movements/min after 1 week of time-out.

Probably the most common procedure for treating psychotic behavior is through the use of drugs. Unfortunately, when drugs are used they not only decrease the rate of the symptoms, but also the rate of nonsymptomatic behavior. As Lindsley (1963) points out, "merely to remove the symptoms, without restoring the nonsymptomatic responding during the psychotic episodes, is in some ways no better than removing the patient's vocal cords or stuffing the attendants' ears with cotton." This is extremely critical when dealing with the mentally retarded, in that to reduce the rate of their nonretarded behavior would be to make them virtually nonfunctional.

Thus, the use of time-out to reduce hallucinations can be not only effective but also an extremely beneficial procedure. It may be that the reduction of such psychotic behavior can be a principal step in the "normalization" process. In a recent survey of a local institution for the retarded, over $50 \%$ of the patients hallucinated at relatively high rates. By applying the systematic analysis of learning theory with clinical experience, one can provide a treatment approach that should help many of the mentally retarded. Hopefully, the reduction of psychotic behavior in these individuals will make their other behavior seem less retarded.

\section{REFERENCES}

Alumbaugh, R. V. Use of behavior modification techniques toward reduction of hallucinatory behavior: A case study. Psychological Record, 1971, 21, 415-417.

Boston, D. E., \& BaIley, J. B. Modification of severe disruptive and aggressive behavior using brief time-out and reinforcement procedures. Journal of Applied Behavior Analysis, 1969, 2, 31-38.

Davidson, G. C. Appraisal of behavior modification techniques with adults in institutional settings. In C. M. Franks (Ed.), Behavior therapy: Appraisal and status. New York: McGraw-Hill, 1969. Pp. 220-278.

HAYNes, S. N., \& GedDY, P. Suppression of psychotic hallucinations through time-out. Behavior Therapy, 1973, 4, 123-127.

LINDSLEY, $O$. Direct measurement and functional definitions of vocal hallucinatory symptoms. Journal of Nervous and Mental Disorders, 1963, 136, 293-297.

REED, G. F. The psychology of anomalous experience: $A$ cognitive approach. London: Hutchinson, 1972.

RutNer, I. T., \& Bugle, C. An experimental procedure for modification of psychotic behavior. Journal of Consulting and Clinical Psychology, 1969, 33, 651-653.

WOLFENSBERGER, W. Will there always be an institution? II: The impact of new service models. Residential alternative to institutions. Mental Retardation, 1971, 10, 21-23.

(Received for publication November 15, 1977.) 